\title{
Predialysis Care and Dialysis Outcomes in Hemodialysis Patients with a Functioning Fistula
}

\author{
Areef Ishani $^{\mathrm{a}-\mathrm{c}}$ David T. Gilbertson ${ }^{\mathrm{c}}$ Deborah Kim ${ }^{\mathrm{d}}$ Brian D. Bradbury ${ }^{\mathrm{d}}$ \\ Allan J. Collins $s^{b, c}$ \\ ${ }^{a}$ Minneapolis VA Health Care System, ${ }^{b}$ Department of Medicine, University of Minnesota, and \\ 'Chronic Disease Research Group, Minneapolis Medical Research Foundation, Minneapolis, Minn., and \\ ${ }^{\mathrm{d} C e n t e r}$ for Observational Research, Amgen, Inc., Thousand Oaks, Calif., USA
}

\section{Key Words}

Care patterns · Epidemiology · Outcomes - Vascular access

\begin{abstract}
Background/Aims: Predialysis care has been associated with improved first-year outcomes. We investigated types of predialysis care associated with improved patient outcomes in patients initiating dialysis with a fistula and at least 2 years of predialysis care. Methods: In this retrospective cohort of incident hemodialysis patients with $\geq 2$ years of Medicare coverage before dialysis initiation, care patterns and patients were determined using Medicare claims. Fistula use at initiation was ascertained from the Medical Evidence Report. Results: Patients aged $\geq 67$ years who initiated hemodialysis with a fistula $(n=14,459)$ differed demographically and clinically from patients who initiated with other vascular access types; however, $55 \%$ had diabetes, $28 \%$ heart failure, and $40 \%$ ischemic heart disease. In the year preceding initiation, $88 \%$ of these patients visited a nephrologist, $66 \%$ a cardiologist, $9 \%$ an endocrinologist, and $3 \%$ a dietician; most underwent routine laboratory measurements. In the first year of dialysis, 50\% were hospitalized and $1.3 \%$ underwent trans-
\end{abstract}

plant; the mortality rate remained constant ( 20 per 100 patient-years). Of predialysis care factors evaluated, only fistula placement more than 1 month before dialysis initiation was associated with lower hospitalization and mortality risk and greater likelihood of transplant. Other potentially modifiable factors included more contact with cardiologists and endocrinologists. Conclusion: Patients initiating dialysis with a functioning fistula appear to receive substantial predialysis preparation. This selected population does not show the excess mortality risk often observed early in dialysis treatment. Earlier fistula placement and referral to cardiology and endocrinology appear to be important aspects of predialysis care.

(c) 2014 S. Karger AG, Basel

\section{Introduction}

The transition to dialysis is a turbulent time marked by high morbidity and mortality. Mortality in the first 120 days is as high as $40 / 100$ patients, and as much as $25 \%$ is due to patient withdrawal $[1,2]$. Surveillance data from the United States Renal Data System indicate that the first-year mortality rate has declined in recent years,

\section{KARGER}

E-Mail karger@karger.com www.karger.com/ajn (c) 2014 S. Karger AG, Basel

0250-8095/14/0393-0238\$39.50/0
Areef Ishani, MD

Chronic Disease Research Group

Minneapolis Medical Research Foundation

914 South 8th Street, Suite S4.100, Minneapolis, MN 55404 (USA)

E-Mail isha0012@umn.edu 
though it remains elevated relative to later years [3]. To meaningfully reduce poor outcomes during the first year after dialysis initiation, patients must receive appropriate care before dialysis initiation; studies suggest that better care in the predialysis period improves outcomes after initiation, although a recent study raised doubt about the strength of that association $[2,4-8]$.

Of the various aspects of care provided to patients with poor kidney function who are approaching dialysis initiation, fistula placement early enough to enable maturation and use at hemodialysis initiation has been most consistently associated with improved outcomes after initiation $[2,9]$. Access to healthcare and appropriate specialist referral has been strongly associated with greater likelihood of initiating dialysis with a fistula $[5,8]$. However, it is unclear whether improved outcomes associated with fistula use are a consequence of patient selection, or if fistula placement is simply a good marker of improved predialysis care.

In a cohort of patients who initiated maintenance hemodialysis with a fistula and who had received at least 2 years of predialysis care, we sought to evaluate the types of predialysis care provided and the aspects of care associated with improved outcomes after initiation. Unlike previous studies focusing almost exclusively on mortality, our analysis also examined all-cause hospitalization and transplant.

\section{Methods}

\section{Data Source and Study Population}

We used Medicare data for patients who initiated hemodialysis between January 1, 2005, and December 31, 2009, with 2 or more years of prior Medicare coverage. To be included in the final cohort, patients were required to be aged $\geq 67$ years at initiation. We required continuous Part A and Part B coverage in the 2 years preceding initiation and a diagnosis of chronic kidney disease (CKD; 2 outpatient claims or 1 inpatient claim) in the $1-2$ years preceding initiation [10]. We required hemodialysis initiation with a functioning fistula, as indicated on the Medical Evidence Report (Centers for Medicare \& Medicaid Services (CMS) form CMS-2728). The date of fistula placement was identified using Medicare claims from the 2 years preceding hemodialysis initiation.

\section{Dimensions of Predialysis Care}

Claims in the year preceding dialysis initiation were reviewed and categorized based on International Classification of Diseases, Ninth Revision, Clinical Modification (ICD-9-CM) diagnosis and procedure codes in the domains imaging and testing (radiology imaging, echocardiograms, biopsies, stress tests), laboratory testing (number of tests for albumin, calcium, creatinine, hemoglobin, hemoglobin $A_{1 c}$, iron panels, parathyroid hormone, phosphorus, urinary protein; laboratory results are not available in
Medicare claims data), outpatient specialist care (number of visits to cardiologists, endocrinologists, nephrologists, radiologists, dieticians, pain specialists), and renal interventions (use, timing, and duration of erythropoiesis-stimulating agents and intravenous iron; number of blood transfusions; vaccinations; timing of fistula placement). We created categories for each of these predialysis care variables to allow balanced subgroups or to differentiate potentially clinically distinct practices based on the distribution of number of visits, administrations, or laboratory tests, as appropriate.

\section{Patient Characteristics at Dialysis Initiation}

Demographic and comorbidity characteristics, laboratory values, and other clinical characteristics assessed at hemodialysis initiation (end-stage renal disease (ESRD) onset factors) were obtained from form CMS-2728 and from claims data in the 1-2 years before dialysis initiation (for comorbidity data). Demographic characteristics included age, sex, race (White, Black, other), and primary cause of ESRD (diabetes, hypertension, glomerulonephritis, other). Comorbid conditions included arrhythmia, cancer, cardiac arrest, cerebrovascular disease, chronic obstructive pulmonary disease, congestive heart failure, diabetes, gastrointestinal bleeding, hypertension, ischemic heart disease, myocardial infarction, and peripheral vascular disease, and number of hospitalizations and mean length of hospital stays. Other clinical characteristics included location of first dialysis session (hospital, nursing home, dialysis center, home), and selected laboratory values (albumin $(\mathrm{g} / \mathrm{dl})$, serum creatinine $(\mathrm{mg} / \mathrm{dl})$, hemoglobin $(\mathrm{g} / \mathrm{dl}))$.

\section{Outcomes}

Outcome events were identified during the year following hemodialysis initiation and included first hospitalization and death or withdrawal from dialysis resulting in death. For the analysis of time to first hospitalization, we followed patients from the date of initiation until the first of date of first inpatient hospitalization event (overnight stay), death, loss to follow-up (transplant, renal recovery), or 1 year. For the analysis of time to death, we followed patients from the date of initiation until the first of date of death, loss to follow-up (transplant, renal recovery) or 1 year. In the case of time to first hospitalization and transfusion, follow-up began at discharge if the patient was hospitalized on the date of ESRD onset. We also explored time to kidney transplant in the first year after dialysis initiation.

\section{Statistical Analysis}

We used descriptive statistics for continuous variables (mean, standard deviation) and categorical variables (count (n), percentage (\%)) to evaluate patient characteristics at dialysis initiation and types of care received before initiation. We did not include $\mathrm{p}$ values in table 1; given the large sample size, all comparisons had a $\mathrm{p}<$ 0.05 . We used Kaplan-Meier methods to estimate the time to event for each outcome of interest. We used Cox proportional hazards regression to estimate hazard ratios (HR) and 95\% confidence intervals (CI) for the unadjusted associations between aspects of predialysis care, demographics, comorbid conditions, and other baseline clinical characteristics and each outcome of interest. We used a backward elimination procedure to arrive at the most parsimonious model for predicting time to hospitalization, transplant, and death. Variables from each of the domains of predialysis care plus 
Table 1. Demographic, comorbidity, and other patient characteristics assessed at dialysis initiation by type of access in use (fistula vs. graft or catheter), in patients with at least 2 years of predialysis care

\begin{tabular}{|c|c|c|}
\hline \multirow[t]{2}{*}{ Characteristic } & \multicolumn{2}{|c|}{ Vascular access } \\
\hline & $\begin{array}{l}\text { fistula } \\
(\mathrm{n}=14,459)\end{array}$ & $\begin{array}{l}\text { graft or } \\
\text { catheter } \\
(n=60,424)\end{array}$ \\
\hline \multicolumn{3}{|l|}{ Demographic } \\
\hline Age, years & $77.0(6.1)$ & $77.8(6.4)$ \\
\hline Female & 37.0 & 49.2 \\
\hline \multicolumn{3}{|l|}{ Race } \\
\hline White & 80.7 & 75.6 \\
\hline Black & 15.6 & 20.7 \\
\hline Asian/other & 3.7 & 3.8 \\
\hline \multicolumn{3}{|l|}{ Cause of ESRD } \\
\hline Diabetes & 41.2 & 45.4 \\
\hline Hypertension & 39.0 & 36.9 \\
\hline Glomerulonephritis & 6.2 & 3.6 \\
\hline Other & 13.0 & 14.1 \\
\hline \multicolumn{3}{|l|}{ Comorbidity } \\
\hline Arrhythmia & 22.2 & 31.9 \\
\hline Cancer & 12.1 & 12.6 \\
\hline Cardiac arrest & 0.2 & 0.5 \\
\hline Cerebrovascular disease & 9.9 & 13.9 \\
\hline Chronic obstructive pulmonary disease & 15.8 & 22.7 \\
\hline Congestive heart failure & 27.8 & 43.2 \\
\hline Diabetes & 55.4 & 63.0 \\
\hline Gastrointestinal bleeding & 3.8 & 7.1 \\
\hline Hypertension & 93.6 & 93.3 \\
\hline Ischemic heart disease & 38.9 & 48.0 \\
\hline Myocardial infarction & 6.8 & 10.3 \\
\hline Peripheral vascular disease & 20.6 & 26.0 \\
\hline \multicolumn{3}{|l|}{ Hospital admissions ${ }^{\mathrm{a}}$} \\
\hline None & 58.5 & 43.2 \\
\hline 1 & 24.6 & 28.2 \\
\hline$\geq 2$ & 16.9 & 28.7 \\
\hline Hospital days ${ }^{a}$ & $9.5(11.0)$ & $13.1(15.3)$ \\
\hline \multicolumn{3}{|l|}{ ESRD onset } \\
\hline \multicolumn{3}{|l|}{ Dialysis setting } \\
\hline Dialysis center or home & 59.2 & 27.2 \\
\hline \multirow{2}{*}{\multicolumn{3}{|c|}{$\begin{array}{l}\text { Hospital or skilled nursing tacillty } \\
\text { Albumin }^{\mathrm{b}}, \mathrm{g} / \mathrm{dl}\end{array}$}} \\
\hline & & \\
\hline$<3.5$ & 43.7 & 67.4 \\
\hline$\geq 3.5$ & 56.3 & 32.6 \\
\hline \multicolumn{3}{|l|}{ Hemoglobin $^{\mathrm{b}}$, g/dl } \\
\hline$<9.0$ & 11.5 & 20.4 \\
\hline $9.0-9.9$ & 20.5 & 25.5 \\
\hline $10.0-10.9$ & 27.8 & 25.9 \\
\hline $11.0-11.9$ & 23.9 & 16.8 \\
\hline$\geq 12.0$ & 16.3 & 11.4 \\
\hline Serum creatinine $\mathrm{e}^{\mathrm{b}}, \mathrm{mg} / \mathrm{dl}$ & $5.5(2.1)$ & $5.3(2.3)$ \\
\hline Estimated $\mathrm{GFR}^{\mathrm{b}}, \mathrm{ml} / \mathrm{min} / 1.73 \mathrm{~m}^{2}$ & $9.8(4.1)$ & $10.6(5.3)$ \\
\hline
\end{tabular}

Values are mean (SD) or percentage. ${ }^{a}$ In patients with $\geq 1$ admission. ${ }^{\mathrm{b}}$ In patients with measured value. demographic and comorbidity characteristics were entered into the model; only those with an associated $\mathrm{p}<0.1$ were retained. Age, sex, and race were forced into all models.

The Hennepin County Medical Center Institutional Review Board approved the analytic plan. All analyses were conducted in SAS 9.2 (Cary, N.C., USA).

\section{Results}

Between 2005 and 2009, 561,232 patients initiated hemodialysis; 14,459 met inclusion criteria and were included in our analysis (fig. 1). On average, patients who initiated hemodialysis with a fistula were more likely to be male (63.0 vs. $50.8 \%)$ and White ( 80.7 vs. $75.6 \%)$, but were similar in age $(77.0 \pm 6.1,77.8 \pm 6.4$ years $)$, compared with patients who initiated with a graft or catheter (table 1). Fistula patients were less likely than graft or catheter patients to have comorbid conditions: arrhythmia (22.2 vs. $31.9 \%)$, chronic obstructive pulmonary disease (15.8 vs. $22.7 \%)$, congestive heart failure ( 27.8 vs. $43.2 \%)$, diabetes (55.4 vs. $63.0 \%$ ), and ischemic heart disease (38.9 vs. $48.0 \%)$.

\section{Care Patterns}

Patients in our study population interacted substantially with the healthcare system in the year preceding dialysis initiation (table 2). Few patients did not undergo outpatient laboratory testing for albumin (4.2\%), calcium $(1.0 \%)$, creatinine $(1.0 \%)$, hemoglobin $(1.1 \%)$, and phosphorus (7.3\%); most patients underwent at least 5 tests for each laboratory measure over the 1-year period. A number of patients did not undergo testing in the preceding year for iron $(28.0 \%)$, parathyroid hormone $(22.2 \%)$, or any quantification of urinary protein excretion (56.4\%). Most patients did not undergo a stress test (77.6\%).

Specialist referral was fairly common. Most patients visited a cardiologist (66.1\%) or a nephrologist (88.1\%) at least once. A substantial percentage $(25.1 \%)$ had visited a nephrologist 13 or more times during the year. Despite the high prevalence of diabetes (55.4\%) and frequent interactions with cardiologists and nephrologists, few patients visited an endocrinologist $(9.4 \%)$ or dietician $(3.3 \%)$ in the year before dialysis initiation.

Fistulas were created 3-11 months before dialysis initiation for $42.4 \%$ of patients and within 1 month of initiation for $2.9 \%$. For $16.3 \%$, the date of access creation could not be ascertained; these were likely placed more than 2 years before initiation, as our claims search went back 2 years, and it is unlikely that a fistula was placed without a Medicare claim being generated. 


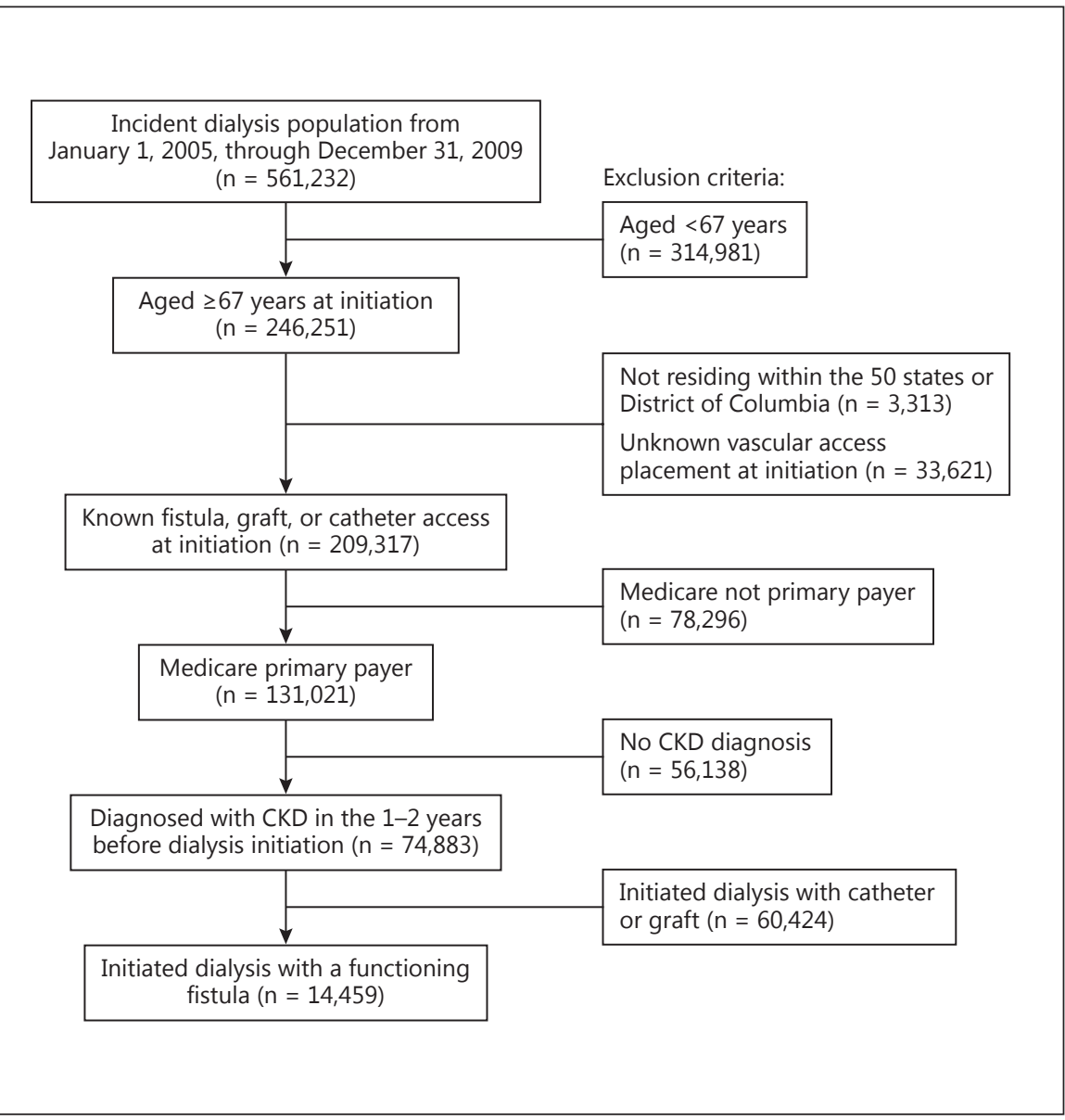

Fig. 1. Cohort selection process.

Medical intervention use was varied during the year preceding hemodialysis initiation. Many patients (37.0\%) received no vaccinations (pneumonia, influenza, or hepatitis B). Hemoglobin concentration was $<10.0 \mathrm{~g} / \mathrm{l}$ for $32.0 \%$ of patients at initiation and albumin was $<3.5 \mathrm{~g} / \mathrm{dl}$ for $43.7 \% ; 19.3 \%$ of patients underwent at least one transfusion. Despite substantial predialysis care, more than $40 \%$ of patients with a fistula initiated hemodialysis in an inpatient setting.

\section{Outcomes}

Nearly $50 \%$ of patients were hospitalized during the first year of hemodialysis, for an incidence rate of 88.1/100 person-years. Median time to first hospitalization was 283 days (fig. 2). A total of 2,631 patients died during the first year, for a mortality rate of 20.0/100 person-years. Notably, mortality rates in the first 120 days and over the entire year were nearly identical (20.4 and 20.0/100 patient-years, respectively).

Outcomes with a Functioning Fistula

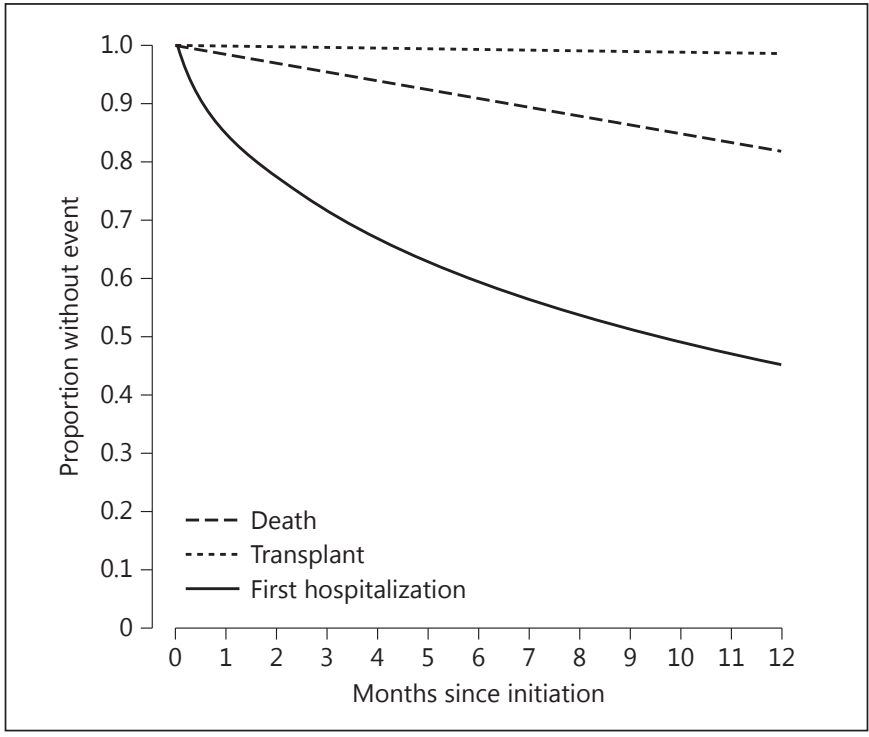

Fig. 2. Kaplan-Meier plots for time to first hospitalization, time to first transplant, and time to mortality during the first year of dialysis among incident dialysis patients initiating with a functioning fistula. 
Table 2. Types of care received in the year preceding initiation of hemodialysis in patients initiating with a functioning fistula $(\mathrm{n}=14,459)$

\begin{tabular}{ll}
\hline Specialist care & \\
Cardiology (visits) & \\
None & 33.9 \\
$1-3$ & 39.9 \\
$\geq 4$ & 26.2 \\
Dietician (1+ visits) & 3.3 \\
Endocrinology (1+ visits) & 9.4 \\
Nephrology (visits) & \\
$\quad$ None & 11.9 \\
$1-6$ & 34.3 \\
$7-12$ & 28.6 \\
$\geq 13$ & 25.1 \\
Pain management (1+ visits) & 1.3 \\
Radiology (visits) & \\
None & 24.9 \\
$1-2$ & 46.1 \\
$\geq 3$ & 29.1 \\
\hline
\end{tabular}

Laboratory tests (tests)

Albumin

None $\quad 4.2$

$1-4 \quad 25.2$

$\geq 5 \quad 70.6$

Calcium

None $\quad 1.0$

$1-5 \quad 10.6$

$\geq 6 \quad 88.4$

Serum creatinine

None $\quad 1.0$

$\begin{array}{ll}1-5 & 8.7\end{array}$

$\geq 6 \quad 90.4$

Hemoglobin

None

$1-5-1.1$

$\geq 6 \quad 83.1$

Hemoglobin $\mathrm{A}_{1 \mathrm{c}}$

None $\quad 48.4$

$1-2 \quad 28.4$

$\geq 3 \quad 23.2$

Iron $^{\mathrm{a}}$

None $\quad 28.0$

$1-2 \quad 34.1$

$\geq 3 \quad 37.9$

Parathyroid hormone

None $\quad 22.2$

$1-2 \quad 32.9$

$\geq 3 \quad 44.9$

Phosphorus

None $\quad 7.3$

$1-3 \quad 22.0$

$\geq 4 \quad 70.6$

Protein electrophoresis (1+ tests) $\quad 12.3$

Urinary protein

None 56.4

$1-2 \quad 29.7$

$\geq 3 \quad 13.8$

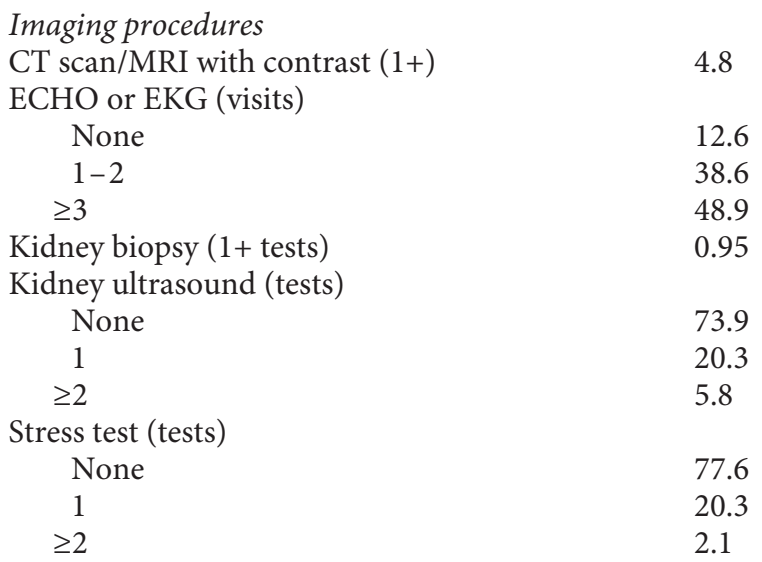

Values are mean $(\mathrm{SD})$ or percentage. $\mathrm{CT}=$ Computed tomography; $\mathrm{ECHO}=$ echocardiography; $\mathrm{EKG}$ = electrocardiogram; ESA = erythropoiesis-stimulating agents; MRI = magnetic resonance imaging.

a Total iron, serum ferritin, or total iron-binding capacity. ${ }^{\mathrm{b}}$ Includes influenza, pneumococcal, and hepatitis. ${ }^{c}$ Before hemodialysis initiation. 
Fig. 3. Unadjusted and multivariable adjusted HR estimates and 95\% CIs for the association between aspects of predialysis care and demographic and comorbidity characteristics and time to (a) first allcause hospitalization event and (b) allcause mortality. $\mathrm{CHF}=$ Congestive heart failure; COPD = chronic obstructive pulmonary disease; CVA/TIA = cerebrovascular accident/transient ischemic attack; $\mathrm{PVD}=$ peripheral vascular disease.

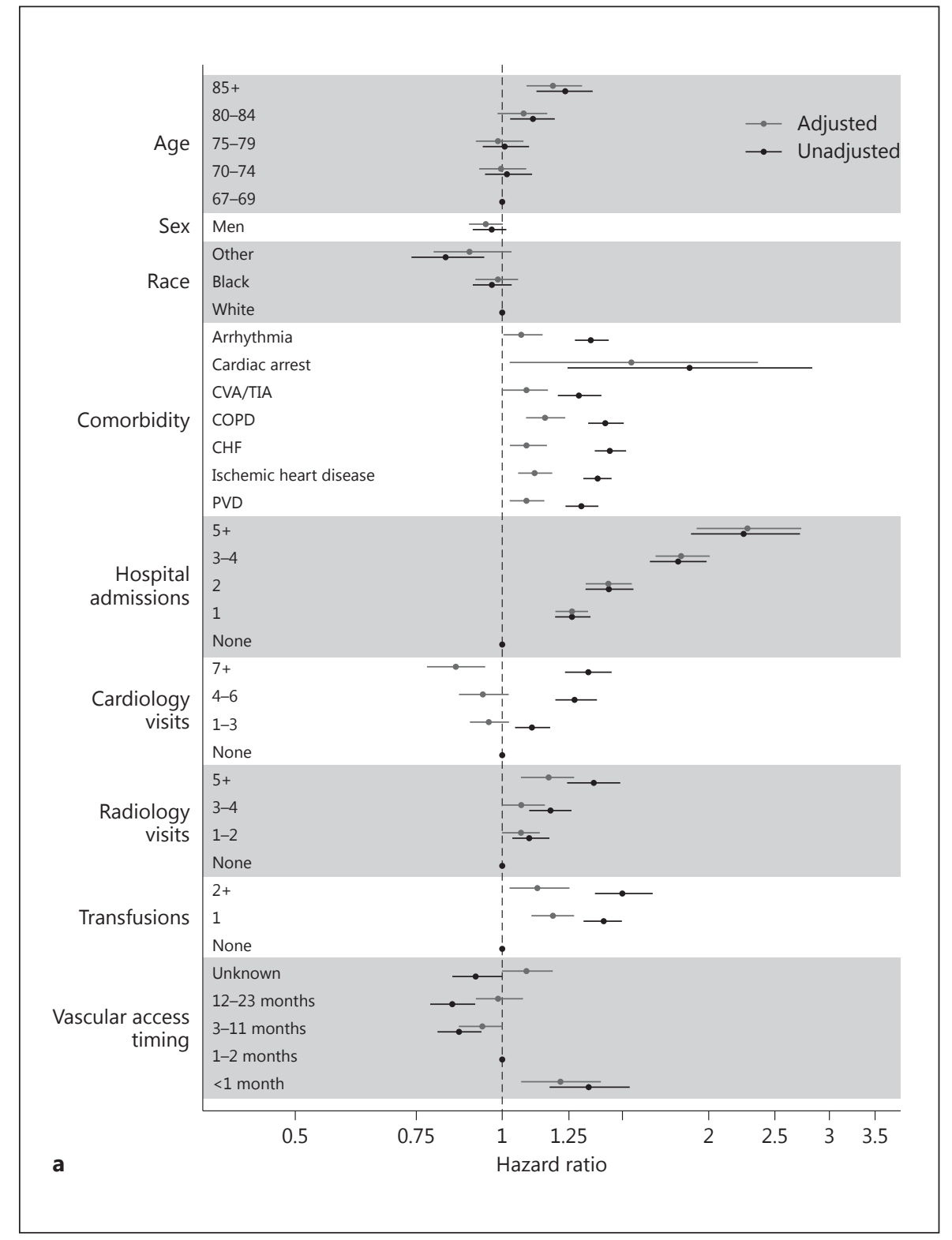

Several factors were associated univariately with time to first hospitalization after initiation. However, in multivariable analysis, only select factors remained strongly associated (fig. 3a). The demographic characteristics age $>85$ years (HR 1.19, 95\% CI 1.09-1.30, reference age 6769 years) and male sex (HR 0.94, 95\% CI 0.90-0.99), and the comorbid conditions arrhythmia (HR 1.07, 95\% CI 1.01-1.13), cerebrovascular disease (HR 1.08, 95\% CI 1.00-1.16), chronic obstructive pulmonary disease (HR $1.15,95 \%$ CI 1.08-1.23), and congestive heart failure (HR $1.09,95 \%$ CI 1.03-1.16) were associated with increased risk.

Outcomes with a Functioning Fistula
Of the interventions evaluated, undergoing more echocardiograms and more laboratory testing (for most parameters assessed) in the year preceding hemodialysis initiation was associated with greater risk of hospitalization following initiation. Of the care patterns evaluated, more frequent visits to a cardiologist in the year preceding initiation was associated with lower risk of hospitalization (HRs 1.0, 0.96, 0.94, 0.85 for $0,1-3,4-6$, and $\geq 7$ visits, respectively; $p$ for linear trend $=0.03$ ). Receiving more transfusions was associated with increased risk of hospitalization. Timing of fistula placement was not associated with hospitalization risk, ex- 


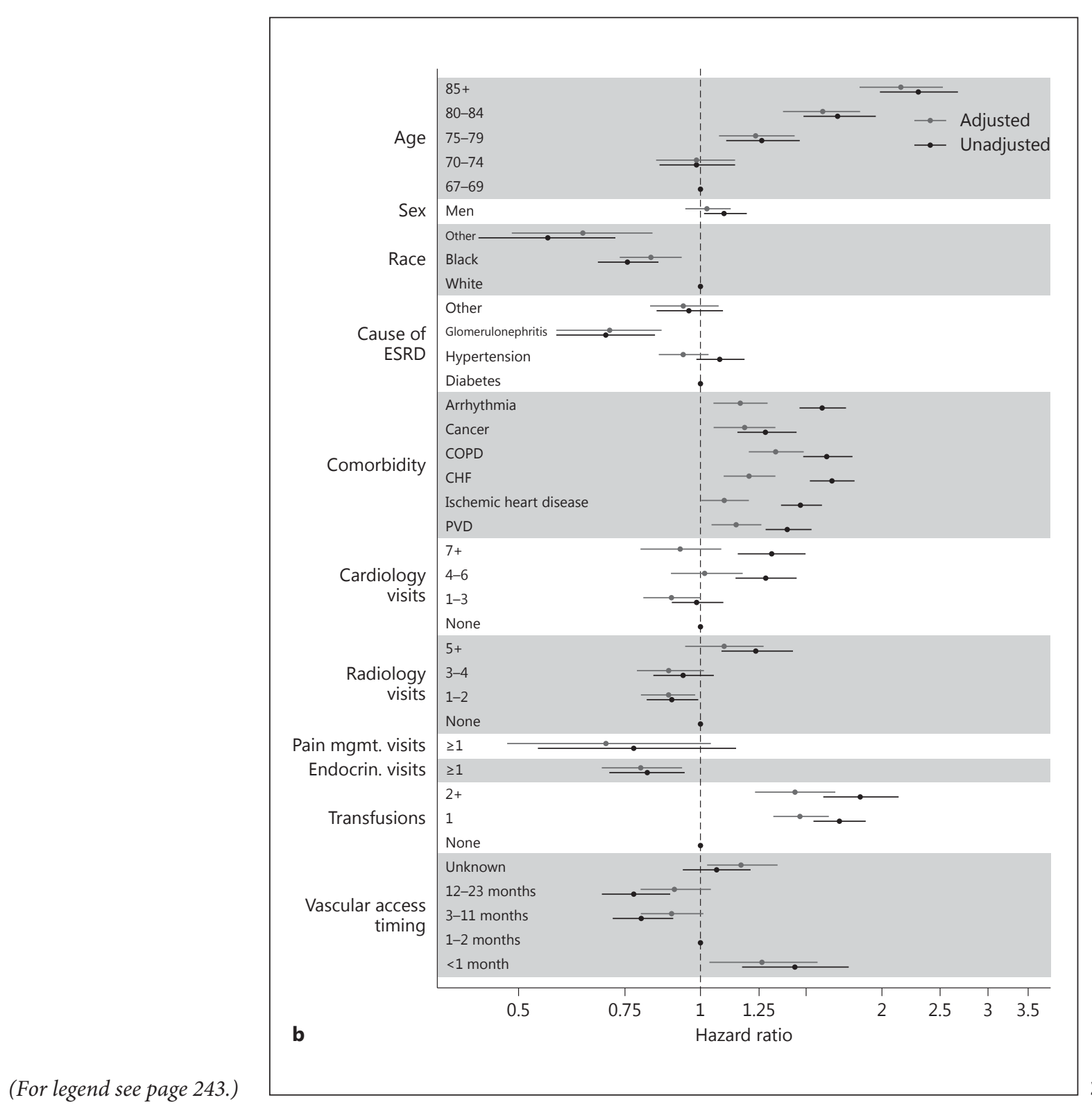

cept for patients with fistulas placed within 1 month before initiation.

Several factors were associated univariately with allcause first-year mortality. However, in multivariable analysis, only select factors remained strongly associated (fig. 3b). We observed similar patterns between frequency of laboratory testing and mortality risk. Of interest were the associations between care patterns observed in the year preceding dialysis initiation and first-year mortality. Visits to an endocrinologist (HR 0.81, 95\% CI 0.69-0.94), but not to a nephrologist or cardiologist, were associated with lower mortality risk. Regarding interventions, num- ber of transfusions was most strongly associated with first-year mortality. Again, fistula placement within 1 month before initiation was associated with increased risk of mortality compared with placement 1-2 months before initiation (HR 1.26, 95\% CI 1.03, 1.55).

Transplant was a relatively infrequent outcome; only 185 patients (1.3\%) underwent transplant during the first year. Several modifiable treatment factors associated with reduced mortality were also associated with an increased odds ratio for transplant, including endocrinologist care, longer time between fistula placement and dialysis initiation, and not receiving a transfusion (table 3 ). 
Table 3. Factors associated with time to transplant among patients initiating dialysis with a functioning fistula

\begin{tabular}{lc}
\hline Characteristics & HR $(95 \%$ CI $)$ \\
\hline Age (per year) & $0.83(0.80-0.86)$ \\
Male & $1.35(0.98-1.87)$ \\
Race (vs. White) & \\
$\quad$ Black & $0.64(0.40-1.03)$ \\
$\quad$ Other & $1.19(0.55-2.57)$ \\
Primary cause of ESRD (vs. diabetes) & \\
$\quad$ Hypertension & $1.57(0.96-2.59)$ \\
$\quad$ Glomerulonephritis & $3.59(2.03-6.37)$ \\
$\quad$ Other & $3.43(2.06-5.71)$ \\
Cerebrovascular disease (yes vs. no) & $0.31(0.11-0.85)$ \\
Ischemic heart disease (yes vs. no) & $0.61(0.41-0.91)$ \\
Endocrinology visit (yes vs. no) & $1.76(1.12-2.78)$ \\
Number of transfusions (vs. 0$)$ & \\
1 & $0.53(0.29-0.97)$ \\
$\geq 2$ & $0.60(0.22-1.62)$ \\
Vascular access placement before ESRD onset, months \\
$\quad<1$ & $1.51(0.43-5.40)$ \\
$\quad 1-2$ & 1.0 \\
$3-11$ & $2.18(1.23-3.87)$ \\
$\geq 12$ & $2.90(1.59-5.27)$ \\
\hline
\end{tabular}

\section{Discussion}

In this study of patients initiating hemodialysis with an optimal vascular access, we identified few modifiable factors in the predialysis period that may be associated with reduced first-year risk of both hospitalization and mortality, and improved likelihood of transplant. The presumption for this study was that patients initiating hemodialysis with a fistula are likely to have an optimal start as they have (1) been identified as having kidney disease early enough to allow vascular access placement with sufficient time for it to mature, and (2) likely seen a nephrologist, as the nephrologist is usually the gatekeeper to vascular access placement. Given these factors, we aimed to determine the overall care provided to these patients and how the care influenced post-initiation outcomes. Despite substantial predialysis care and presence of a functioning fistula, $40.8 \%$ of patients initiated hemodialysis in an inpatient setting, a potential area of opportunity. An additional important observation was that the mortality rate remained relatively constant throughout the first year. We did not observe an initial spike in the rate as has been previously observed $[1,2]$, suggesting that high mortality in the first few months on dialysis may be largely attributable to poor preparation [3]. Our results also suggest that despite optimal vascular access at dialysis ini-

Outcomes with a Functioning Fistula tiation, patients initiating with a fistula did not achieve guideline concordance on a number of factors.

Numerous studies, including a recent systematic review [11], have suggested that improved predialysis care is associated with improved outcomes in ESRD patients. However, most previous studies included patients who began dialysis emergently with little preparation and likely high risk of adverse outcomes. Our study aimed to identify a cohort likely to have received predialysis renal care (i.e. patients starting dialysis with a functioning fistula) to minimize selection bias. Compared with the general incident dialysis population, patients initiating with a fistula were clinically different and were more likely to have seen a nephrologist in the preceding year. This finding is not unexpected; in most circumstances, the nephrologist is the gateway to dialysis fistula placement, and more nephrology visits has been associated with greater likelihood of a functioning fistula at dialysis initiation [12-14]. Patients in our cohort had a substantial number of outpatient nephrology visits; more than $50 \%$ had more than seven visits in the year preceding dialysis initiation. Most surprisingly, despite having a functioning fistula at dialysis initiation, $11.9 \%$ of patients had not seen a nephrologist in the preceding 2 years. Given the significant number of nephrologist visits in the preceding year, it is surprising that fistula placement occurred well in advance of dialysis initiation. Access was placed within 1 month before dialysis initiation for only $3 \%$ of patients. A short time between fistula placement and dialysis initiation was associated with increased risk of hospitalizations and mortality, and with reduced odds of transplant. Our results do not clarify how a short time between fistula placement and poor outcomes is mediated. It may reflect the importance of routine, long-term care before dialysis initiation, which would enable earlier fistula placement. Alternatively, use of a fistula early after its creation may lead to early fistula failure, resulting in subsequent catheter placement; hence, the adverse outcomes we observed may result from catheter placement [15]. However, an analysis of DOPPS data did not suggest a greater risk of fistula failure associated with early cannulation $[15,16]$.

Nearly all patients (96.7\%) had not visited a dietician before dialysis initiation. Our results are consistent with our previous analysis evaluating dietician visits reported on form CMS-2728 [17]. That analysis found a similarly low percentage (12\%) of ESRD patients seeing a dietician in the year preceding dialysis initiation. Compared with patients who did not see a dietician in the predialysis period, those who did were more likely to have higher albumin and lower total cholesterol concentrations at dialysis 
initiation. In our current study, despite optimal dialysis access and significant predialysis exposure to nephrologists, albumin concentration was below current guidelines $(3.5 \mathrm{~g} / \mathrm{dl})$ for more than $40 \%$ of patients. We are unaware of any randomized controlled trial evaluating the merits of predialysis dietician care, but given the substantial nutritional restrictions placed on CKD and ESRD patients, including dietary counseling as part of a comprehensive approach to CKD seems prudent.

Anemia management appears to offer another opportunity for improvement in care. Hemoglobin concentration was $<9.0 \mathrm{~g} / \mathrm{l}$ for $11.5 \%$ of patients who initiated dialysis with a fistula, and $19.3 \%$ of patients received at least one outpatient transfusion in the year preceding initiation. Transfusions in the predialysis period were associated with increased risk of hospitalization and of mortality. In this study, we did not differentiate between transfusions administered to treat acute bleeds or traumatic events from transfusions administered to manage chronic anemia; thus, these results may simply reflect worse underlying prognosis. We also observed that likelihood of undergoing transplant was lower for patients who received a predialysis transfusion. This result is in keeping with a previous study suggesting that blood transfusions in CKD are associated with a $28 \%$ decrease in the odds of transplant [18]. Our finding may reflect elevations in panel reactive antibody levels in pretransplant patients who receive blood transfusions $[19,20]$, although we cannot exclude the possibility that it simply reflects a better underlying clinical profile for patients who do not receive transfusions.

Our study is limited in several ways. First, many of our predictor and covariate variables are from claims data. Claims data have been shown to be specific for the item of interest but to lack sensitivity [21]. In addition, although we attempted to characterize and evaluate more intensive care (as measured by more frequent visits or tests), we could not differentiate between better care and greater need for care. Thus, some of our estimates (e.g. receiving more laboratory tests) likely represent greater disease severity and need for more intensive clinical management. Our primary requirement for cohort entry was presence of a functioning fistula at hemodialysis initiation. This information was derived from form CMS-2728, and its accuracy is unknown; however, we expect that it has high specificity as this information is easily ascertained in the dialysis unit. Also, few patients in this elderly cohort underwent transplant, and therefore our results may not be generalizable to the broader incident dialysis population. Our cohort was chosen to identify care and outcomes of patients destined to initiate hemodialysis. Given this pre- condition, finding a population to which our results are immediately applicable is difficult. Our results can thus only be considered hypothesis-generating.

Our study also has some strengths. We used the entire cohort of older patients initiating hemodialysis in the United States with a functioning fistula. We attempted to adjust our results for several factors present in the 2 years preceding initiation. Finally, we limited our study population to patients with documented predialysis care, as evidenced by initiating dialysis with an optimal access, in order to reduce the potential for residual confounding. This approach differs from previous investigations that included in the reference group patients who initiated dialysis emergently, often without receiving care from general practitioners or specialists.

Patients who initiate hemodialysis with a functioning fistula are in general well cared for in the predialysis period, though opportunities for improvement exist. Among the predialysis care factors evaluated, earlier placement of a fistula before dialysis initiation and more specialist care, particularly by cardiologists and endocrinologists, appear to be important modifiable factors that may positively affect risk of hospitalization and mortality and likelihood of undergoing transplant after dialysis initiation. Other potential improvements include better nutritional management and minimizing the number of dialysis initiations in the inpatient setting. Lastly, unlike in the overall incident population, we did not observe an increase in mortality risk immediately following dialysis initiation. Future studies should investigate whether this is due to better access to care and appropriate preparation in the predialysis period or simply reflects a healthier population initiating dialysis.

\section{Acknowledgements}

This study was supported by a research contract with Amgen, Inc., Thousand Oaks, Calif., USA. The authors thank Chronic Disease Research Group colleagues Delaney Berrini, BS, for manuscript preparation, and Nan Booth, MSW, MPH, ELS, for manuscript editing.

\section{Disclosure Statement}

Before submission for peer review, the manuscript was reviewed by the sponsor. Comments were sent to the authors, who are solely responsible for the final version. The analysis, interpretation, and reporting of these data are the responsibility of the authors. Dr. Ishani reports no conflicts of interest. Dr. Gilbertson has provided consultation to Amgen, DaVita Clinical Research, and Affymax. Ms. Kim and Dr. Bradbury work in the Center for Observational Research at Amgen, Inc. Dr. Collins has provided consultation to NxStage, Affymax/Takeda, and Amgen. 


\section{References}

1 United States Renal Data System. USRDS 2010 Annual Data Report: Atlas of Chronic Kidney Disease and End-Stage Renal Disease in the United States. Bethesda, National Institutes of Health, National Institute of Diabetes and Digestive and Kidney Diseases, 2010.

-2 Bradbury BD, Fissell RB, Albert JM, et al: Predictors of early mortality among incident US hemodialysis patients in the Dialysis Outcomes and Practice Patterns Study (DOPPS) Clin J Am Soc Nephrol 2007;2:89-99.

3 United States Renal Data System. USRDS 2012 Annual Data Report: Atlas of Chronic Kidney Disease and End-Stage Renal Disease in the United States. Bethesda, National Institutes of Health, National Institute of Diabetes and Digestive and Kidney Diseases, 2012.

-4 Biesenbach G, Hubmann R, Janko O, Schmekal B, Eichbauer-Sturm G: Predialysis management and predictors for early mortality in uremic patients who die within one year after initiation of dialysis therapy. Ren Fail 2002; 24:197-205.

$\checkmark 5$ Winkelmayer WC, Owen WF Jr, Levin R, Avorn J: A propensity analysis of late versus early nephrologist referral and mortality on dialysis. J Am Soc Nephrol 2003;14:486-492.

6 Khan SS, Xue JL, Kazmi WH, et al: Does predialysis nephrology care influence patient survival after initiation of dialysis? Kidney Int 2005;67:1038-1046.
7 Avorn J, Bohn RL, Levy E, et al: Nephrologist care and mortality in patients with chronic renal insufficiency. Arch Intern Med 2002;162: 2002-2006.

$\checkmark 8$ Smart NA, Titus TT: Outcomes of early versus late nephrology referral in chronic kidney disease: a systematic review. Am J Med 2011; 124:1073-1080 e2.

-9 Winkelmayer WC, Liu J, Chertow GM, Tamura MK: Predialysis nephrology care of older patients approaching end-stage renal disease. Arch Intern Med 2011;171:13711378 .

10 Hebert PL, Geiss LS, Tierney EF, Engelgau MM, Yawn BP, McBean AM: Identifying persons with diabetes using Medicare claims data. Am J Med Qual 1999;14:270-277.

11 Black C, Sharma P, Scotland G, et al: Early referral strategies for management of people with markers of renal disease: a systematic review of the evidence of clinical effectiveness, cost-effectiveness and economic analysis. Health Technol Assess 2010;14:1-184.

12 Stehman-Breen CO, Sherrard DJ, Gillen D, Caps M: Determinants of type and timing of initial permanent hemodialysis vascular access. Kidney Int 2000;57:639-645.

13 Astor BC, Eustace JA, Powe NR, et al: Timing of nephrologist referral and arteriovenous access use: the CHOICE Study. Am J Kidney Dis 2001;38:494-501.

-14 Avorn J, Winkelmayer WC, Bohn RL, et al: Delayed nephrologist referral and inadequate vascular access in patients with advanced chronic kidney failure. J Clin Epidemiol 2002; 55:711-716.
15 Bradbury BD, Chen F, Furniss A, et al: Conversion of vascular access type among incident hemodialysis patients: description and association with mortality. Am J Kidney Dis 2009;53:804-814.

16 Saran R, Dykstra DM, Pisoni RL, et al: Timing of first cannulation and vascular access failure in haemodialysis: an analysis of practice patterns at dialysis facilities in the DOPPS. Nephrol Dial Transplant 2004;19:2334-2340.

17 Slinin Y, Guo H, Gilbertson DT, et al: Prehemodialysis care by dietitians and first-year mortality after initiation of hemodialysis. Am J Kidney Dis 2011;58:583-590.

18 Ibrahim HN, Skeans MA, Li Q, Ishani A, Snyder JJ: Blood transfusions in kidney transplant candidates are common and associated with adverse outcomes. Clin Transplant 2011;25: 653-659.

19 Balasubramaniam GS, Morris M, Gupta A, Mesa IR, Thuraisingham R, Ashman N: Allosensitization rate of male patients awaiting first kidney grafts after leuko-depleted blood transfusion. Transplantation 2012;93:418422.

20 Yabu JM, Anderson MW, Kim D, et al: Sensitization from transfusion in patients awaiting primary kidney transplant. Nephrol Dial Transplant 2013;28:2908-2918.

21 Grams ME, Plantinga LC, Hedgeman E, et al: Validation of CKD and related conditions in existing data sets: a systematic review. Am J Kidney Dis 2011;57:44-54. 